\title{
RAGE-mediated inflammation, type 2 diabetes, and diabetic vascular complication
}

\section{Yasuhiko Yamamoto* and Hiroshi Yamamoto}

Department of Biochemistry and Molecular Vascular Biology, Kanazawa University Graduate School of Medical Sciences, Kanazawa, Japan

Edited by:

Tsuguhito Ota, Kanazawa University, Japan

Reviewed by:

Undurti Narasimha Das, UND Life

Sciences, USA

Chul-Hee Kim, Soonchunhyang

University, South Korea

\section{*Correspondence:}

Yasuhiko Yamamoto, Department of Biochemistry and Molecular Vascular Biology, Kanazawa University

Graduate School of Medical Sciences,

13-1 Takara-machi, Kanazawa

920-8640, Japan

e-mail: yasuyama@med.kanazawa-

u.ac.jp
Obesity is associated with inflammation and type 2 diabetes. Innate immune system comprised of cellular and molecular components plays an important role in the inflammatory reactions. Immune cells like macrophages and their cell surface pattern-recognition receptors (PRRs) are representative for innate immunity promoting inflammatory reactions. The receptor for advanced glycation end-products (RAGE) is a member of PRRs and a proinflammatory molecular device that mediates danger signals to the body. The expression of RAGE is observed in adipocytes as well as immune cells, endothelial cells, and pancreatic $\beta$ cells under certain conditions. It has been reported that RAGE is implicated in adipocyte hypertrophy and insulin resistance. RAGE-mediated regulation of adiposity and inflammation may attribute to type 2 diabetes and diabetic vascular complications.

Keywords: rage, obesity, inflammation, toll-like receptors, pattern-recognition receptors
Obesity is associated with an increased risk of developing type 2 diabetes, fatty liver disease, hypertension, and vascular complications (1). Proinflammatory and anti-inflammatory bioactive molecules produced from adipose tissues, known as adipokines, contribute to the burden of obesity-related diseases (2). Adipose tissue consists of heterogeneous populations of adipocytes, stromal preadipocytes, immune cells, and vascular cells, and it can respond rapidly and dynamically to alterations in nutrient excess caused by enhanced food consumption through adipocyte hypertrophy and hyperplasia (3). This results in a local inflammation in adipose tissue that propagates an overall systemic but chronic lowgrade inflammation associated with the development of obesityrelated comorbidities such as type 2 diabetes and cardiovascular diseases (2).

\section{INNATE IMMUNITY AND RAGE-MEDIATED INFLAMMATORY REACTIONS}

The innate immune system can act as a double-edged sword in protecting the host against foreign enemies and destroying tissues via inflammation. It may represent an evolutionary strategy adopted by multicellular organisms to prevent the survival of cells that would otherwise cause more disastrous consequences in the individuals and their descendants. Toll-like receptors (TLR) and receptor for advanced glycation end-products (RAGE) can participate in innate immunity maintaining a delicate balance between clearance of pathogens and induction of exaggerated inflammatory responses.

Receptor for advanced glycation end-products is originally identified for recognizing advanced glycation end-products (AGE) (4). RAGE belongs to the immunoglobulin superfamily, and is now known as a member of pattern-recognition receptors
(PRRs) and as a proinflammatory device. RAGE recognizes a variety of endogenous and exogenous ligands, including AGE, advanced oxidation protein products, high-mobility group box protein 1 (HMGB1), calcium-binding $S 100$ proteins, $\beta 2$-integrin Mac-1/CD11b, amyloid $\beta$ peptide/fibril, lipopolysaccharide (LPS), phosphatidylserine, C1q, and lysophosphatidic acid (LPA) (5). It has been hypothesized that RAGE engagement of such ligands causes diabetic vascular complications, atherosclerosis, cancer, neurodegeneration, and inflammatory diseases (6). Anti-RAGE antibody treatment is reported to suppress lung metastasis of cancer cells and to offer a survival advantage to septic mice $(7,8)$. Downstream intracellular signaling molecules of RAGE include NFKB, ERK (extracellular signal-regulated kinase) 1/2, p38MAPK (mitogen-activated protein kinases), JNK (c-Jun Nterminal kinases), PKC (protein kinase C), Rac/Cdc42, and TIRAP and MyD88, adaptor proteins for TLR 2 and 4 (9). A functional link between RAGE and TLR is thus considered to be in a coordinated manner (10).

Among the above ligands, HMGB1 is known to be readily released from necrotic or damaged cells and to be actively secreted by activated endothelial cells and immune cells such as monocytes, macrophages, dendritic cells, and natural killer cells (11). HMGB1 can form a complex with proinflammatory molecules of CpG DNA, LPS, and interleukin $1 \beta$, and this further induces the activation of RAGE signaling (12). HMGB1 is also found to be expressed in human adipose tissues with the expression levels associated with the fat mass and obesity-related genes (13). TLR2 and 4 also recognize HMGB1 and can be involved in HMGB1-induced cellular responses (14). S100 proteins are a family of over 20 proteins that show a structural similarity with their two EF-hand $\mathrm{Ca}^{2+}$-binding domains flanked by $\alpha$-helices. Higher oligomerization states of S100 proteins lead to the activation of RAGE (15). 
AGE-modified S100A8/A9 have been reported to strongly activate inflammatory responses via RAGE (16). S100A8/A9 was also shown to interact with TLR4 (17). Our groups have also shown that phosphatidylserine on the surface of apoptotic cells and LPS are also RAGE ligands $(18,19)$. Rapid removal of apoptotic cells by phagocytes is crucial for tissue development, homeostasis, resolution of inflammation, and prevention of autoimmune responses. RAGE was found to function as one of the PS receptors that recognize and initiate apoptotic cell clearance (18). LPS and the lipid A component responsible for LPS toxicity and known as endotoxin were found to directly interact with RAGE (19). LPS is also a well-known TLR ligand.

\section{RAGE AND ADIPOSITY}

Using RAGE and apoE double deficient mice, Ueno et al. demonstrated that absence of RAGE is associated with decreased epididymal fat weight and smaller adipocyte size, which are significantly associated with the decrease in atherosclerotic lesions (20). They also reported that circulating anti-inflammatory adiponectin levels in apoE $^{-1-} \mathrm{RAGE}^{-1-}$ were higher than apoE ${ }^{-/-} \mathrm{RAGE}^{+/+}$ mice, and their levels were significantly and inversely associated with aortic atherosclerosis. Very recently, Monden et al. demonstrated that RAGE directly regulated adipogenesis and hypertrophic process of adipocyte differentiation in vitro (21). Adenoviral overexpression of RAGE markedly increased generation of hypertrophic adipocytes and RAGE knockdown by using siRNA system significantly suppressed generation of hypertrophic adipocytes. Under high fat diet feeding in mice, RAGE deficiency is associated with less body weight, less epididymal fat weight, less adipocyte size, higher serum adiponectin, higher expressions of Glut4 and adiponectin in epididymal fat, and greater insulin sensitivity. It is now acceptable that direct role of RAGE in adipocyte hypertrophy and insulin resistance (Figure 1). However, RAGE ligands are still unknown to be involved in the RAGE-dependent adiposity. Further studies are required to characterize the interplays among a variety of RAGE ligands and inflammatory reactions in obesity and type 2 diabetes.

\section{REFERENCES}

1. Romeo GR, Lee J, Shoelson SE. Metabolic syndrome, insulin resistance, and roles of inflammation - mechanisms and therapeutic targets. Arterioscler Thromb Vasc Biol (2012) 32:1771-6. doi:10.1161/ ATVBAHA.111.241869

2. Donath MY, Shoelson SE. Type 2 diabetes as an inflammatory disease. Nat RevImmunol (2011) 11:98-107. doi:10.1038/nri2925

3. Shoelson SE, Lee J, Goldfine $\mathrm{AB}$. Inflammation and insulin resistance. $J$ Clin Invest (2006) 116:1793-801. doi:10.1172/JCI29069

4. Neeper M, Schmidt AM, Brett J, Yan SD, Wang F, Pan YC, et al. Cloning and expression of a cell surface receptor for advanced glycosylation end products of proteins. J Biol Chem (1992) 267:14998-5004.

5. Yamamoto Y, Yamamoto H. and diabetic vascular complications. J Diabetes Invest (2011) 2: 155-7. doi:10.1111/j.2040-1124. 2011.00125.x

6. Yan SF, Yan SD, Ramasamy R, Schmidt AM. Tempering the wrath of RAGE: an emerging therapeutic strategy against diabetic complications, neurodegeneration, and inflammation. Ann Med (2009) 25:1-15. doi:10.1080/07853890902806576

7. Christaki E, Opal SM, Keith JC Jr, Kessimian N, Palardy JE, Parejo NA, et al. A monoclonal antibody against RAGE-mediated inflammation

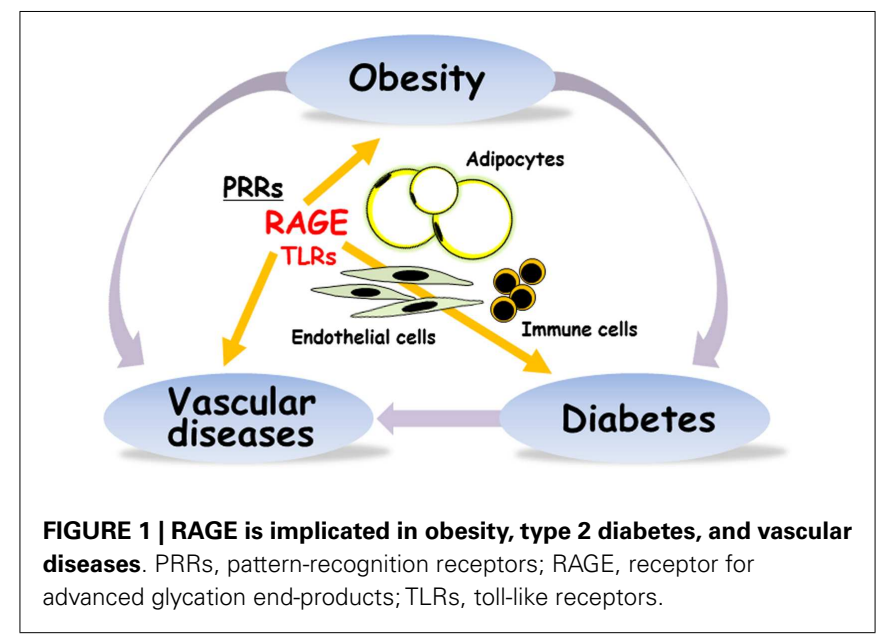

\section{RAGE POLYMORPHISMS, OBESITY, AND INFLAMMATION}

Several functional single nucleotide polymorphisms have been identified in human RAGE gene. The G82S occurs in the ligand-binding $\mathrm{V}$ domain of RAGE and affects ligand affinity, resulting in the enhancement of proinflammatory reactions and immune/inflammatory diseases $(22,23)$. In obese subjects, S/S carriers showed significantly higher concentrations of AGE and $C$ reactive protein than $G$ allele carrier and lower concentration of soluble RAGE, a decoy receptor (24). S allele at RAGE G82S polymorphism may be more closely associated with proinflammatory reactions under obese conditions rather than nonobese status, thus linking to the development of obesity-associated complications.

We very recently reported that the induction of RAGE expression in pancreatic $\beta$-cell by insufficient leptin action under obesity conditions could trigger $\beta$-cell failure in type 2 diabetes (25). It is thus considered that RAGE could be a potential targeting receptor for the prevention and treatment of the development of obesity, $\beta$-cell failure, vascular complications, and inflammation in type 2 diabetes (Figure 1).

RAGE alters gene expression and is protective in experimental models of sepsis and pneumococcal pneumonia. Shock (2011) 35:492-8. doi: 10.1097/SHK.0b013e31820b2e1c

8. Mizumoto S, Takahashi J, Sugahara K. Receptor for advanced glycation end products (RAGE) functions as receptor for specific sulfated glycosaminoglycans, and anti-RAGE antibody or sulfated glycosaminoglycans delivered in vivo inhibit pulmonary metastasis of tumor cells. J Biol Chem (2012) 287:18985-94. doi:10.1074/jbc.M111.313437

9. Yamamoto Y, Yamamoto H. Controlling RAGE to conquer diabetic vascular complications. J Diabetes Invest (2012) 3:107-14. doi:10. 1111/j.2040-1124.2011.00191.x
10. Sakaguchi M, Murata H, Yamamoto K, Ono T, Sakaguchi Y, Motoyama A, et al. TIRAP, an adaptor protein for TLR2/4, transduces a signal from RAGE phosphorylated upon ligand binding. PLoS ONE (2011) 6:e23132. doi:10.1371/ journal.pone.0023132

11. Tsan MF. Heat shock proteins and high mobility group box 1 protein lack cytokine function. J Leukoc Biol (2011) 89:847-53. doi:10.1189/jlb. 0810471

12. Tian J, Avalos AM, Mao SY, Chen B, Senthil K, Wu H, et al. Tolllike receptor 9-dependent activation by DNA-containing immune complexes is mediated by HMGB1 and RAGE. Nat Immunol (2007) 8:48796. doi:10.1038/ni1457 
13. Qin YH, Dai SM, Tang GS, Zhang J, Ren D, Wang ZW, et al. HMGB1 enhances the proinflammatory activity of lipopolysaccharide by promoting the phosphorylation of MAPK p38 through receptor for advanced glycation end products. J Immunol (2009) 183:6244-50. doi:10.4049/jimmunol.0900390

14. Park JS, Svetkauskaite D, He Q, Kim JY, Strassheim D, Ishizaka A, et al. Involvement of toll-like receptors 2 and 4 in cellular activation by high mobility group box 1 protein. J Biol Chem (2004) 279: 7370-7. doi:10.1074/jbc. M306793200

15. Leclerc E, Fritz G, Vetter SW, Heizmann CW. Binding of S100 proteins to RAGE: an update. Biochim Biophys Acta (2009) 1793: 993-1007. doi:10.1016/j.bbamcr. 2008.11.016

16. Turovskaya O, Foell D, Sinha P, Vogl T, Newlin R, Nayak J, et al. RAGE, carboxylated glycans and S100A8/A9 play essential roles in colitis-associated carcinogenesis. Carcinogenesis (2008) 29:2035-43. doi:10.1093/carcin/bgn 188

17. Vogl T, Tenbrock K, Ludwig S, Leukert N, Ehrhardt C, van Zoelen MA, et al. Mrp8 and Mrp14 are endogenous activators of Toll-like receptor 4, promoting lethal, endotoxin-induced shock. Nat Med (2007) 13:1042-9. doi:10.1038/nm1638

18. He M, Kubo H, Morimoto K, Fujino N, Suzuki T, Takahasi T, et al. Receptor for advanced glycation end products binds to phosphatidylserine and assists in the clearance of apoptotic cells. $E M B O$ Rep (2011) 12:358-64. doi:10.1038/ embor.2011.28

19. Yamamoto Y, Harashima A, Saito $\mathrm{H}$, Tsuneyama K, Munesue S, Motoyoshi S, et al. Septic shock is associated with receptor for advanced glycation end products ligation of LPS. J Immunol (2011) 186:3248-57. doi:10.4049/ jimmunol.1002253

20. Ueno H, Koyama H, Shoji T, Monden M, Fukumoto S, Tanaka S, et al. Receptor for advanced glycation end-products (RAGE) regulation of adiposity and adiponectin is associated with atherogenesis in apoE-deficient mouse. Atherosclerosis (2000) 211:431-6. doi:10.1016/j. atherosclerosis.2010.04.006

21. Monden M, Koyama H, Otsuka Y, Morioka T, Mori K, Shoji T, et al. Receptor for advanced glycation end products regulates adipocyte hypertrophy and insulin sensitivity in mice: involvement of Toll-like receptor 2. Diabetes (2013) 62:47889. doi:10.2337/db11-1116

22. Osawa M, Yamamoto Y, Munesue S, Murakami N, Sakurai S, Watanabe $\mathrm{T}$, et al. De-N-glycosylation or G82S mutation of RAGE sensitizes its interaction with advanced glycation endproducts. Biochim Biophys Acta (2007) 1770 1468-74. doi:10.1016/j.bbagen. 2007.07.003

23. Han Z, Liu Q, Sun C, Li Y. The interaction between obesity and RAGE polymorphisms on the risk of knee osteoarthritis in Chinese population. Cell Physiol Biochem (2012) 30:898-904. doi:10.1159/ 000341467

24. Kim OY, Jo SH, Jang Y, Chae JS, Kim JY, Hyun YJ, et al. G allele at RAGE SNP82 is associated with proinflammatory markers in obese subjects. Nutr Res (2009) 29:106-13. doi:10. 1016/j.nutres.2009.01.006

25. Han D, Yamamoto Y, Munesue S, Motoyoshi S, Saito H, Win MT, et al. Induction of receptor for advanced glycation end products by insufficient leptin action triggers pancreatic $\beta$-cell failure in type 2 diabetes.
Genes Cells (2013) 18:302-14. doi: $10.1111 /$ gtc. 12036

Conflict of Interest Statement: The authors declare that the research was conducted in the absence of any commercial or financial relationships that could be construed as a potential conflict of interest.

Received: 09 April 2013; accepted: 06 August 2013; published online: 21 August 2013.

Citation: Yamamoto Y and Yamamoto $H$ (2013) RAGE-mediated inflammation, type 2 diabetes, and diabetic vascular complication. Front. Endocrinol. 4:105. doi: 10.3389/fendo.2013.00105

This article was submitted to Diabetes, a section of the journal Frontiers in Endocrinology.

Copyright (c) 2013 Yamamoto and Yamamoto. This is an open-access article distributed under the terms of the Creative Commons Attribution License (CC $B Y)$. The use, distribution or reproduction in other forums is permitted, provided the original author(s) or licensor are credited and that the original publication in this journal is cited, in accordance with accepted academic practice. No use, distribution or reproduction is permitted which does not comply with these terms. 